\title{
En los vértices del tiempo. Metáforas conceptuales del tiempo y sus variaciones en la poesía y el pensamiento filosófico
}

In the vertices of time. Conceptual metaphors of time and its variations in poetry and philosophical thinking

ENRIQUE HUELVA UNTERNBÄUMEN

UNIVERSIDAD DE BRASILIA

Artículo recibido el / Article received: 2019-02-17

Artículo aceptado el / Article accepted: 2019-10-02

RESUMEN: En este trabajo pretendemos analizar la relación que pudiera existir entre la metáfora conceptual general TIEMPO ES ESPACIO (Lakoff y Johnson, 1999; Raden, 2003; Núñez, 2003; Ellen, 2016) que utilizamos para la conceptualización cotidiana de la temporalidad y conceptualizaciones del tiempo y la temporalidad elaboradas en el ámbito del pensamiento filosófico y la producción poética. Específicamente, pretendemos averiguar hasta qué punto y de qué forma los conceptos cotidianos influencian nuestras reconceptualizaciones estéticas y filosóficas. $O$, al revés: en qué medida éstas últimas consiguen liberarse de las primeras. En concreto, analizaremos el concepto fenomenológico de la temporalidad, como propuesto por Edmund Husserl (2002) y Merleau-Ponty (1945), así como algunas elaboraciones poéticas presentes en poemas de Jorge Manrique, Goethe, Unamuno, Antonio Machado, Chico Buarque, entre otros. Como principales resultados podemos anticipar, por una parte, un profundo enraizamiento de las propuestas estéticas y filosóficas en la metáfora general del cotidiano. Por otra, no obstante, queda evidenciado también que las metáforas generales sirven de espacio fuente para reconceptualizaciones que pueden alcanzar un alto grado de complejidad cognitiva y semántica. Estas reconceptualizaciones son producidas por los mismos mecanismos y procesos responsables por la variación intercultural de las metáforas conceptuales (Kövecses, 2005, 2008).

Palabras clave: metáforas temporales; variación metafórica; poesía; filosofía; blending.

ABSTRACT: In this paper, we intend to analyze the relationships that may exist between the conceptual metaphor TIME IS SPACE (Lakoff y Johnson, 1999; Raden, 2003; Núñez, 2003; Ellen, 2016) that we use for everyday conceptualization of temporality, on the one hand, and the conceptions of time and of temporality drawn up within the spheres of philosophical thought and poetry, on the other. Specifically, it is our intention to verify the extent to which everyday concepts influence our aesthetic and philosophical conceptualizations; or, viceversa, the degree to which the latter are able to free themselves from the former. 
Concretely, we shall analyze the phenomenological concept of temporality, as proposed by Edmund Husserl (2002) and Merleau-Ponty (1945), alongside certain poetical theses present in the poems of Jorge Manrique, Goethe, Unamuno, Antonio Machado and Chico Buarque, among others. We anticipate that the principal results will, on the one hand, be to show that the underpinnings of the aesthetic and philosophical proposals are more deeply rooted in the general everyday metaphors. Nevertheless, we will also present evidence that the general metaphors serve as a source space for reconceptualizations that may attain a high degree of cognitive and semantic complexity. These reconceptualizations are produced by the same mechanisms and processes responsible for the intercultural variation of conceptual metaphors (Kövecses, 2005, 2008).

Key words: time metaphors; metaphorical variation; poetry; philosophy; blending.

\section{INTRODUCCIÓN}

Mais s'il est un état où l'ame trouve une assiette assez solide pour s'y reposer tout entiere $\&$ rassembler là tout son être, sans avoir besoin de rappeler le passé, ni d'enjamber sur l'avenir; où le tans ne soit rien pour elle, où le présent dure toujours sans néanmoins marquer sa durée \& sans aucune trace de succession, sans aucun autre sentiment de privation ni de jouissance, de plaisir ni de peine, de désir ni de crainte que celui seul de notre existence, \& que ce sentiment seul puisse la remplir tout entiere; tant que cet état dure, celui qui s'y trouve peut s'appeler heureux, non d'un bonheur imparfait, pauvre \& relatif tel que celui qu'on trouve dans les plaisirs de la vie; mais d'un bonheur suffisant, parfait \& plein, qui ne laisse dans l'ame aucun vide qu'elle sente le besoin de remplir. Tel est l'état où je me suis trouvé souvent à l'Isle de St. Pierre dans mes rêveries solitaires, soit couché dans mon bateau que je laissois dériver au gré de l'eau, soit assis sur les rives du lac agité, soit ailleurs au bord d'une belle riviere ou d'un ruisseau murmurant sur le gravier. De quoi jouit-on dans une pareille situation? De rien d'extérieur à soi, de rien sinon de soimême \& de sa propre existence; tant que cet état dure, on se suffit à soi-même comme Dieu.

Jean-Jacques Rousseau ${ }^{1}$

Desde el ruiseñor que encantó a Ruth en los trigales de Belén de Judá hasta la percepción abstracta de la madreselva y del barro fundamental, como puros hechos homogéneos, que describe Borges en su Historia de la eternidad (2005: 40-44), no son, ni mucho menos, escasos los relatos que nos sugieren que la eternidad (o la atemporalidad), o sea la supresión de la sucesión, de la ordenación fundamental en un antes y un después -aquel «tiempo sin tiempo» anhelado por Benedetti- es una forma posible de nuestra

\footnotetext{
${ }^{1}$ Traducción del autor: Pero si hay un estado en el que el alma encuentra un acomodo lo bastante sólido como para descansar en él por entero y congregar todo su ser, sin tener necesidad de recordar el pasado ni exceder del porvenir, donde el tiempo no existe para ella, donde el presente dure siempre, sin señalar, no obstante, su duración y sin huella alguna de secuencia, sin ningún otro sentimiento de goce, de placer o de dolor, de deseo o de temor que el de nuestra existencia, y que este sentimiento único pueda colmarla por entero; en tanto dura tal estado, quien se encuentre en él puede llamarse dichoso, no de una dicha imperfecta, pobre y relativa, tal cual se halla en los placeres de la vida, sino de una dicha suficiente, perfecta y plena que no deja en el alma ningún vacío que ésta sienta la necesidad de llenar. Tal es el estado en que me encontré con frecuencia en la isla Saint-Pierre en mis ensoñaciones solitarias, ora tumbado en mi barca que dejaba derivar a merced del agua, ora sentado en las riberas del lago agitado, ora en otra parte, a orillas de un hermoso río o de un arroyo murmurando por entre el guijarral. ¿De qué se goza en semejante situación? De nada externo a uno, de nada sino de uno mismo y de su propia existencia; en tanto tal estado dura uno se basta a sí mismo como Dios. [Traducción del autor].
} 
experiencia. Prácticas espirituales o de contemplación mística, momentos extásicos en rituales religiosos, o la experiencia estética de lo sublime, corroboran, sin duda, que es razonable postular la existencia de esta forma de relacionarnos con las cosas y con nosotros mismos.

Sin embargo, no es menos atinado puntualizar que se trata, en estos y en otros casos semejantes, de actividades y circunstancias que se alejan -cada una a su manerade la forma más normal que tenemos de experimentar el tiempo. Como bien observa Niklas Luhmann (1995: 107-109) en sus comentarios sobre las ensoñaciones del solitario Rousseau, la renuncia a la temporalidad, o más concretamente, al pasado y al porvenir, con la consecuencia de un "presente que dura para siempre», no es algo que simplemente acaece, sino que es el resultado de una acción específica, a saber, de la «acción concentrada que tiene como finalidad desactivar toda acción». ${ }^{2}$ Lo mismo puede ser aseverado con respecto a cualquiera de las otras prácticas y experiencias que acabo de mencionar.

En efecto, sin la intervención de una de estas acciones concentradas, nuestro cotidiano parece estar acompasado ineludiblemente por la temporalidad, por la disposición de nuestras experiencias en algún tipo de sucesión, en alguna fórmula que genere un antes y un después. En este sentido, Merleau-Ponty (1945: 471) inicia el capítulo dedicado a la temporalidad en la Fenomenología de la percepción, refiriéndose a la misma como «el carácter más general de nuestros hechos psíquicos».

La sucesión de ideas y pensamientos y la temporalidad resultante tiene un correlato en la comunicación, especialmente manifiesto en aquella generada en su forma más básica: la de la interacción comunicativa. La interacción está basada en eventos, en turnos de habla, en enunciados, en actos de habla iniciales y actos de habla reactivos, que a medida que son producidos dan origen a la realización de otros, percibidos como posteriores, creando así una temporalidad propia que organiza y estructura la actividad comunicativa. Sin pretender extenderme mucho en su análisis, valga una observación sobre la naturaleza de la temporalidad comunicativa. Como señala Fuchs en su estudio de la «máquina de comunicación» (1995: 13-46), el evento comunicativo causa un presente postergado o dislocado, en el sentido de que el enunciado que acaba de ser proferido - y que, por lo tanto, ya no existe más en su materialidad - es el enunciado actualmente presente. Con cada nuevo enunciado se reproduce esta postergación del presente.

En suma, parece difícil escapar de la temporalidad como desafío principal para la conceptuación de nuestra experiencia del tiempo. Y esto vale tanto para los conceptos cotidianos, como para los que desarrollamos en las reflexiones filosóficas o en los procesamientos estéticos.

En este trabajo intentaremos elucidar justamente la relación que pudiera existir entre estos dos niveles de conceptualización de la temporalidad. La principal pregunta que se plantea es si los conceptos fílosóficos y estéticos pueden ser analizados como variaciones (lingüísticas) de los cotidianos o, si, por el contrario, la creatividad en estos ámbitos es capaz de trascender los moldes de las metáforas del cotidiano y crear estructuras conceptuales diferentes. En concreto, analizaremos el concepto fenomenológico de la temporalidad, como propuesto por Merleau-Ponty, así como algunas elaboraciones literarias presentes en poemas de Jorge Manrique, Goethe, Unamuno, Antonio Machado, Chico Buarque, entre otros. Hemos seleccionado autores y obras pertenecientes al ámbito cultural occidental, escritos en diversas lenguas europeas y que poseen, como una de sus principales características, la conceptualización estética de la temporalidad. Pero antes de iniciar esta labor, precisamos primero introducir

\footnotetext{
${ }^{2}$ Merece mención especial también el análisis de las Ensoñaciones de Rousseau que nos ofrece Peter Slotedijk en el ensayo Stress und Freiheit (2011).
} 
brevemente las características fundamentales de la conceptualización cotidiana (cultural) de la temporalidad.

\section{LA CONCEPTUALIZACIÓN COTIDIANA DE LA TEMPORALIDAD}

Para circunscribir y caracterizar adecuadamente la conceptualización de la temporalidad es importante diferenciarla de nuestras experiencias fenomenológicas de la sucesión de elementos (eventos, procesos, etc.). De un modo general, podemos afirmar que estas experiencias sirven de base (que podríamos denominar proto-temporalidad) sobre la cual operan procesos de conceptualización.

Las culturas y lenguas del mundo presentan diferencias, pero también semejanzas significativas en relación con qué aspectos de esta base experimental son seleccionados prioritariamente para funcionar como punto de partida de procesos de conceptualización de la temporalidad. Ellen (2016) identifica cuatro modelos básicos utilizados por las diversas culturas del mundo: el modelo geofísico, el medioambiental, el social y el corporal. En muchos casos, las culturas utilizan, al mismo tiempo, más de uno de ellos, incluso de forma combinada.

Son frecuentes, por ejemplo, conceptualizaciones de la temporalidad resultantes de la interrelación entre el movimiento de cuerpos celestes (modelo geofísico) y la secuencia típica de actividades ganaderas y agrícolas (siembra, cosecha, movimientos de trashumancia del pastoreo, etc.), pertenecientes al modelo medioambiental. Los ciclos que se van originando a partir de esta interrelación nos ofrecen, según este autor (2016: 130-131), un medio para modelar el paso del tiempo, tanto en periodos de tiempo cortos (dentro de un mismo año), como en los más extensos (superiores al año).

La conceptualización de temporalidad puede basarse también en eventos o acciones sociales, como, por ejemplo, ritos o festividades religiosas o ritos iniciáticos o de pasaje, como la pubertad, el matrimonio, el nacimiento, los ritos fúnebres, etc. (Ellen, 2016: 132134).

Y, finalmente, nuestro propio cuerpo (o, mejor dicho: nuestra propiocepción) nos ofrece una fuente rica - aunque bastante diversa - de datos que nutren a la elaboración de una noción o, por lo menos, de una percepción de temporalidad. Si los ordenamos según la duración de los intervalos, tendremos: (i) el registro de la sucesión en el procesamiento de informaciones y percepciones, el pulso, la respiración, etc. (los llamados ritmos ultradianos); (ii) la distribución de comidas, descanso/sueño versus actividad a lo largo de un día (ritmos circadianos), y (iii) ciclos y trasformaciones con ocurrencias temporales más dilatadas (ritmos infradianos), como el ciclo menstrual o las diferentes etapas del desarrollo del cuerpo del ser humano (Ellen, 2016: 134-136).

Esta diversidad de modelos y sus combinaciones no deben ser, sin embargo, óbice para reconocer el hecho de que existe un denominador común, que subyace transversalmente a todos ellos, a saber, la experiencia fenomenológica de una sucesión de elementos (eventos) que se van ordenando en un antes y un después, sea conformando una linealidad $(\mathrm{A}>\mathrm{B}>\mathrm{C} \ldots$ ), sea configurando un ciclo $(\mathrm{A}>\mathrm{B}>\mathrm{A}>\mathrm{B} \ldots)$. $\mathrm{Y}$, como dijimos más arriba, es esta experiencia básica la que constituye el punto de partida (el dominio fuente) para procesos de conceptualización de la temporalidad.

Como sucede también en el caso de otras experiencias abstractas, para conceptuar la temporalidad nos valemos, en gran medida, del mecanismo de la metáfora conceptual (Lakoff y Johnson, 1980, 1999; Lakoff, 1993; Kövecses 2010, 2017; Ellen, 2016: 140). Para caracterizar cómo funciona este mecanismo en el caso concreto de la temporalidad, es necesario que llevemos en cuenta tres aspectos fundamentales (Huelva Unternbäumen, 2013: 8-19): (i) nuestro esquema corporal; (ii) la intercorporeidad y (iii) nuestras 
experiencias corpóreas en el espacio, especialmente nuestros movimientos en él. Veamos cada uno de ellos.

(i) El esquema corporal hace referencia a nuestras formas básicas de ser, estar y actuar en el mundo como seres dotados de una determinada corporeidad constituida por un conjunto de capacidades táctiles, auditivas, visuales, proprioceptivas, kinestésicas, etc. (Merleau-Ponty, 1945: 17; Depraz, 2001: 172-173). Un aspecto esencial del esquema corporal es lo que Harald Weinrich (2001: 18-21) denomina asimetrías, a saber, la distribución desigual de nuestros órganos y capacidades sensoriales, perceptivas y comunicativas por el cuerpo. La primera de ellas es la asimetría de la frontalidad, que hace referencia al hecho de que estos órganos y capacidades se concentran en la parte anterior del cuerpo. Además, estos órganos y sus respectivas capacidades también están distribuidos de forma asimétrica a lo largo del eje vertical del cuerpo, con una marcada predominancia de la parte superior, especialmente de la cabeza. Weinrich (2001: 20) denomina esta asimetría asimetría vertical. Finalmente, existe también una asimetría lateral, que reside en el hecho de que nuestra «mano buena»-la derecha para los diestros y la izquierda para los zurdos- suele asumir con mayor frecuencia la ejecución de acciones, inclusive tareas de gesticulación e indicación durante la interacción comunicativa.

(ii) No sólo tenemos el mismo esquema corporal, sino que además sabemos que lo tenemos. La identificación de que el Otro tiene un cuerpo como el mío, que posee las mismas asimetrías, representa un aspecto de la intersubjetividad de gran importancia para la conceptualización por medio del lenguaje y para la comunicación. Siguiendo la tradición fenomenológica, llamaremos a este conocimiento compartido intercorporeidad Depraz (2001: 171-174). La intercorporeidad constituye un prerrequisito básico para la comunicación, puesto que el alineamiento corporal, característico de una situación de interacción comunicativa, se lleva a cabo en forma de una frontalidad coordinada (Weinrich 2001: 19), lo que presupone que previamente debemos haber sido capaces de identificar de forma recíproca que nuestros cuerpos comparten esta característica.

(iii) La intercorporeidad es, asimismo, un elemento esencial de la percepción y de la conceptualización lingüística. Mucho de lo que está codificado por la gramática remite directamente o indirectamente a ella. Así, como está ampliamente documentado en los estudios sobre gramaticalización, un gran número de preposiciones espaciales en las lenguas del mundo derivan, por procesos de gramaticalización, de lexemas que designan partes del cuerpo (Heine y Kuteva, 2001). El uso de estas preposiciones activa como un presupuesto básico para su comprensión aspectos de nuestra intercorporeidad. La comprensión, por ejemplo, de ante en la oración No pudo contener la emoción y se desmayó ante el féretro de su esposa supone una frontalidad del sujeto (esposo) con relación al objeto (féretro). Esto es, el cuerpo del sujeto no está solo delante del féretro, sino también 'girado' hacia él. Al mismo tiempo, tenemos que llamar la atención sobre una frontalidad más implícita, concerniente al propio sujeto-conceptuador: quien utiliza este enunciado asume también una posición frontal con respecto a la totalidad de la escena descrita. Del mismo modo, el movimiento expresado en la oración Los excursionistas caminaron hacia la ermita supone una frontalidad de los excursionistas con relación a su destino y, igualmente, una frontalidad implícita del sujeto-conceptuador con relación a la escena observada.

La importancia del esquema corporal compartido no se restringe a la conceptuación de relaciones espaciales, sino que se adentra, como proyección metafórica, en otros dominios de nuestra experiencia. Es lo que ocurre en el caso de la temporalidad, conceptualizada mediante la metáfora TIEMPO ES ESPACIO (Lakoff y Johnson, 1999: 137169; Raden, 2003; Núñez, 2003; Kövecses, 2010: 37-38). 
Esta metáfora da origen a tres modelos cognitivos básicos. El primer modelo incluye un sujeto experimentador que ocupa una determinada posición en el espacio que equivale al momento presente, a su «ahora». El sujeto permanece estático. Por el contrario, los elementos temporales (momentos, eventos, acontecimientos, etc.) son concebidos como objetos en movimiento, objetos que se dirigen al sujeto, que están junto a él o que ya lo han pasado y se encuentran detrás del mismo. Es gracias a este movimiento que entendemos 'el pasar del tiempo' y creamos una división temporal que incluye un futuro (objetos que están delante del sujeto, todavía 'por venir'), un presente (objetos que están junto al sujeto) y un pasado (objetos que están detrás del sujeto). La figura 1 ilustra este primer modelo.

Figura 1: tiempo como objetos en movimiento.

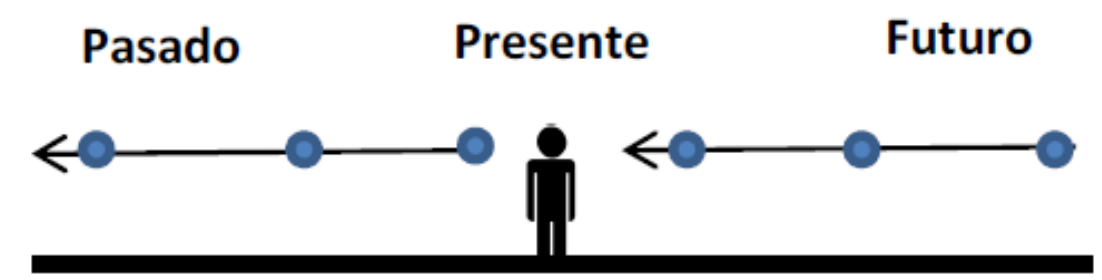

En el segundo modelo se invierte esta relación y ahora los elementos temporales son estáticos, conceptuados como una sucesión de objetos que ocupan posiciones fijas en el espacio, a lo largo de la cual se va moviendo un sujeto experimentador dinámico. Los objetos que todavía se encuentran delante del sujeto representan el futuro, los que va dejando atrás el pasado y los que se encuentran a su misma altura el presente. En la figura 2 representamos este modelo.

Figura 2: tiempo como movimiento del sujeto

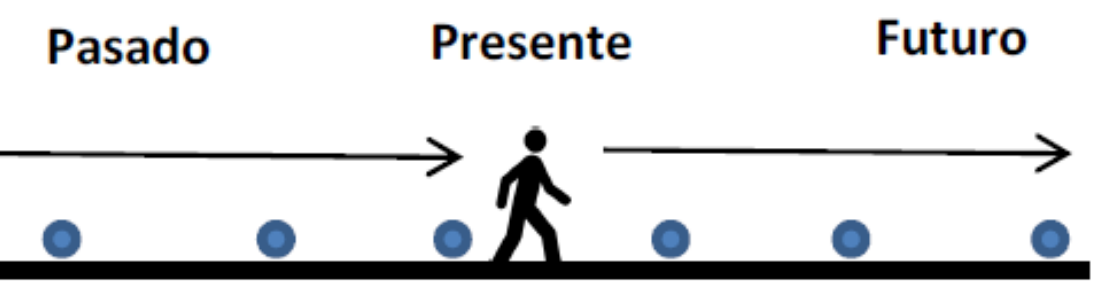

El tercer y último modelo se diferencia de los dos anteriores en que no incluye un sujeto experimentador explícito. Está formado tan solo por un conjunto de objetos distribuidos de forma fija y estática en el espacio. Este modelo nos ayuda a conceptuar un elemento temporal como anterior con respecto a otro elemento temporal y se manifiesta en enunciados como, por ejemplo: el lunes precede al martes, Juan viene antes de 
Navidad, etc. La figura 3 corresponde a este modelo.

Figura 3: tiempo como una secuencia de objetos

Después

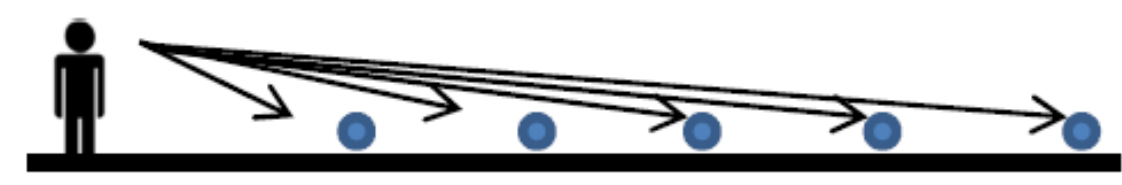

Ahora bien, es importante observar que para que este modelo funcione es necesario determinar si el orden se establece observando los objetos de la derecha hacia la izquierda o al contrario de la izquierda hacia la derecha. Dicho de otro modo: es necesaria la presencia de un observador. En consecuencia, tenemos aquí también -aunque de una forma más sutil que en los otros dos modelos- la actuación de un sujeto experimentador.

Si comparamos con atención los modelos que acabamos de presentar, constataremos que, a pesar de las diferencias específicas que hemos señalado, tienen en común una característica esencial: los tres se fundamentan en la frontalidad perceptiva del sujeto experimentador. Para conceptuar el tiempo como una serie de objetos que se mueven en dirección al sujeto, éste tiene que estar necesariamente 'frente a ellos', para percibir que se están aproximando, que están aquí o que ya han pasado. Lo mismo ocurre si es él el que se mueve en dirección a los objetos o si partimos de la perspectiva de un observador implícito que establece un orden relativo en un conjunto de objetos estáticos. Si llevamos esto en consideración, hemos de puntualizar que el espacio al cual se refiere la metáfora conceptual general TIEMPO ES ESPACIO es el espacio percibido y conceptuado bajo las condiciones que impone nuestro esquema corporal, especialmente su frontalidad perceptiva. Lo mismo puede ser afirmado con respecto al movimiento de la metáfora EL TIEMPO COMO MOVIMIENTO (MOVING TIME METAPHOR) (Lakoff y Johnson, 1999: 141-144; Kövecses, 2010: 37) o de la metáfora EL TIEMPO COMO MOVIMIENTO DEL OBSERVADOR (MOVING OBSERVER METAPHOR) (Lakoff y Johnson, 1999: 146-148; Kövecses, 2010: 38). En suma, la metáfora incorpora la frontalidad perceptiva, como elemento central de nuestro esquema corporal, a la experimentación y conceptuación de la temporalidad.

Para finalizar, es necesario que hagamos algunas ponderaciones sobre la universalidad intercultural de lo que acabamos de exponer. La primera y más amplia, tiene que ver con la propia metáfora TIEMPO ES ESPACIO. Los datos de los que disponemos hasta el momento sobre la conceptualización de la temporalidad en las lenguas y culturas del mundo nos obligan a adoptar una cierta cautela y a hablar más bien de una casi universalidad, en lugar de una universalidad absoluta de esta metáfora. Así, por ejemplo, Sampaio et al. (2011) señalan que en amondawa (lengua hablada en el estado de Rondonia, perteneciente a la familia Tupi-Kawahib) no es frecuente el uso de palabras con sentido de localización espacial o movimiento para expresar temporalidad. A esta relativización general, hay que añadir, además, otras que apuntan hacia una variabilidad cultural significativa en la concretización de esta metáfora general. Es el caso del aimara (y de otras lenguas americanas y africanas), lengua en la que se produce una inversión del alineamiento detrás-pasado versus delante-futuro (Núñez y Sweetser, 2006). Como conjunto de hechos conocidos, el pasado es concebido como lo accesible y, por lo tanto, como aquello que está frente a mí, dispuesto ante mis órganos perceptivos. El futuro, en su calidad de lo desconocido, permanece inaccesible a mi percepción $y$, 
consecuentemente, detrás de mí. Observemos, pues, que el aimara mantiene la estructura fundamental de la metáfora TIEMPO ES ESPACIO, incluyendo la asimetría de la frontalidad como constituyente corpóreo inherente a ella, pero permuta los elementos de los mappings, los elementos del dominio del tiempo conectados a la dualidad resultante de esta asimetría: el futuro pasa a estar conectado a los objetos no percibidos del espacio, mientras que el pasado se conecta a los objetos percibidos en él. Una variación distinta en el alineamiento la encontramos en mandarín - para dar un último ejemplo. En esta lengua, existe una asociación de antes con superior (o más arriba) y, consecuentemente, de después con inferior (o más abajo), como se puede percibir en los ejemplos siguientes:

(1) Shang-ban-tian

Superior-mitad-día

Mañana

(2) Xia-ban-tian

Inferior-mitad-dia

(Yu, 1998: 67)

Según Shimohara (2000), la motivación para este alineamiento la hemos de buscar en la percepción del descenso de objetos o seres vivos por pendientes. Vemos antes el objeto en la parte superior que en la inferior. Este alineamiento del mandarín conserva, por lo tanto, la estructura fundamental de la metáfora TIEMPO ES ESPACIO, bien como la asimetría de la frontalidad perceptiva, cambiando, no obstante, el tipo de percepción en el dominio del movimiento.

\section{LOS MOVIMIENTOS DEL TIEMPO EN LA POESÍA Y EN LA FENOMENOLOGÍA FILOSÓFICA}

Iniciamos esta sección con algunas consideraciones teórico-metodológicas importantes para desarrollar el análisis. Volvamos, en primer lugar, al objetivo central de este trabajo e intentemos concretarlo a la luz de lo expuesto en la sección anterior. La pregunta central puede ser ahora reformulada del modo siguiente: ¿hasta qué punto y de qué forma la poesía y la filosofía se sirven de las metáforas del cotidiano para conceptualizar nuestras experiencias de la temporalidad (nuestra proto-temporalidad)? De un modo general, las obras que se han ocupado hasta el momento de la relación entre metáforas conceptuales y poesía sugieren que la creatividad poética afecta más a las formas lingüísticas por las que se expresan las metáforas que a la estructura conceptual de las mismas (Lakoff y Turner, 1989; Kövecses, 2018). En este sentido, Lakoff y Turner (1989: 1-56) señalan que los poetas comparten con los otros individuos las mismas metáforas y que este hecho explicaría que usen exactamente estas metáforas del cotidiano para hacerse entender en sus poesías:

Poets must make the most of the linguistic and conceptual resources they are given. Basic metaphors are part of those conceptual resources, part of the way members of our culture make sense of the world. Poets may compose ore elaborate or express them in new ways, but they still use the same basic conceptual resources available to us all. If they did not, we would not understand them.

(Lakoff y Turner, 1989: 26) ${ }^{3}$

\footnotetext{
${ }^{3}$ Traducción del autor: Los poetas deben aprovechar al máximo los recursos lingüísticos y conceptuales que poseen. Las metáforas básicas son parte de esos recursos conceptuales, parte de la forma en que los miembros de nuestra cultura le dan sentido al mundo. Los poetas pueden componerlas, elaborarlas o
} 
Los ejemplos que analizamos a continuación muestran, sin embargo, que la creatividad poética y fillosófica puede trascender el nivel lingüístico y transformar la estructura conceptual de las metáforas del cotidiano. La poesía y la reflexión filosófica van más allá de la elaboración de nuevas formas lingüísticas para expresar las mismas metáforas. Y también van más allá de una mera especificación de metáforas genéricas o de la combinación de metáforas simples (e inalteradas) para crear otras más complejas, como proponen Lakoff y Turner (1989: 67-72). La creación poética y filosófica en relación con el tiempo y la temporalidad es, en gran medida, también un proceso de creación metafórica. En este proceso, las metáforas del cotidiano desempeñan la función de punto de partida - de espacio fuente, para expresarlo más técnicamente - para la (re)conceptualización poética o filosófica de nuestras experiencias temporales, de nuestra proto-temporalidad.

Añadamos a estas ponderaciones preliminares una última de carácter más metodológico. La construcción metafórica en los poemas y los escritos filosóficos analizados se lleva a cabo en tres niveles distintos. Encontramos, en primer lugar, palabras usadas metafóricamente, como, por ejemplo, 'navegar' con el significado metafórico de 'guiar el destino de la vida', 'camino' con el significado metafórico de 'vida', etc. El segundo nivel corresponde al empleo de figuras literarias propias de los textos poéticos, como la metáfora poética, el símil o la comparación, para la expresión de metáforas conceptuales (Kövecses, 2018). Es lo que sucede en casos como 'Nuestras vidas son los ríos...', que expresa la metáfora conceptual general LA VIDA ES UNA TRAVESÍA o en 'El tiempo se precipita como una piedra desde lo alto de una montaña' que remite a la metáfora EL TIEMPO ES UN MOVIMIENTO IMPREVISIBLE. El tercer y último nivel es el del sentido general del texto. En la famosa fórmula machadiana 'Hoy es siempre todavía', ninguna de las palabras, tomadas aisladamente, tiene uso metafórico. Tampoco se emplea en ella una figura literaria como las que acabamos de mencionar. Aun así, el sentido general de la expresión transforma significativamente la estructura conceptual de las dos metáforas generales TIEMPO COMO MOVIMIENTO y TIEMPO COMO MOVIMIENTO DEL OBSERVADOR, como demostraremos más adelante.

De estas observaciones hemos de concluir que no podemos privilegiar en nuestro análisis metodologías del tipo 'bottom-up', que se centren únicamente en la detección del uso metafórico de palabras, como propuesto por el Pragglejaz Group (2007). Por lo menos tan importante como este abordaje, es un análisis de la construcción metafórica global a partir del sentido general del texto o partes de él. En síntesis, es aconsejable -como sugiere Kövecses (2011) - una combinación de metodologías, en la que procedimientos 'bottom-up' y 'top-down' se complementen. Es lo que intentaremos desarrollar a continuación.

Empecemos por el caso más simple. No son pocos los ejemplos que adoptan de forma inalterada o casi inalterada la metáfora general TIEMPO ES ESPACIO, corroborando, en este aspecto, la visión propuesta por Lakoff y Turner (1989). Que «el tiempo pasa y nos vamos poniendo viejos» (Pablo Milanés), pero también que «lo nuestro es pasar» (Antonio Machado), lo sabemos por lo menos desde las Coplas a la muerte de su padre, de Jorge Manrique:

$$
\text { I }
$$

Recuerde el alma dormida, avive el seso y despierte contemplando

expresarlas de nuevas maneras, pero continúan usando los mismos recursos conceptuales básicos disponibles para todos nosotros. Si no lo hicieran, no los entenderíamos. 
II

cómo se pasa la vida, cómo se viene la muerte tan callando, cuán presto se va el placer, cómo después, de acordado, da dolor; cómo, a nuestro parecer, cualquiera tiempo pasado fue mejor.

$\begin{array}{ll} & \text { Pues si vemos lo presente } \\ \text { cómo en un punto se es ido } \\ \text { y acabado, } \\ \text { si juzgamos sabiamente, } \\ \text { daremos lo no venido } \\ \text { por pasado. } \\ \text { No se engañe nadie, no, } \\ \text { pensando que ha de durar } \\ \text { lo que espera, } \\ \text { más que duró lo que vio } \\ \text { porque todo ha de pasar } \\ \text { por tal manera. } \\ \text { Nuestras vidas son los ríos } \\ \text { que van a dar en la mar, } \\ \text { que es el morir; } \\ \text { allí van los señoríos } \\ \text { derechos a se acabar } \\ \text { y consumir; } \\ \text { allí los ríos caudales, } \\ \text { allí los otros medianos } \\ \text { y más chicos, } \\ \text { y llegados, son iguales } \\ \text { los que viven por sus manos } \\ \text { y los ricos. } \\ \text { (...) }\end{array}$

Las coplas conceptualizan la temporalidad recurriendo a las dos metáforas dinámicas, a saber, TIEMPO COMO MOVIMIENTO y TIEMPO COMO MOVIMIENTO DEL OBSERVADOR (sujeto). Así, por una parte, la vida pasa, la muerte viene y el placer se va, pero por otra, nuestras vidas son los ríos, que trazan su curso desde el nacimiento hasta la desembocadura en el mar, que es el morir.

La referencia al curso de un río es, sin duda, una de las concretizaciones poéticas más frecuentes de la metáfora TIEMPO COMO MOVIMIENTO DEL OBSERVADOR y, seguramente, también su origen. Sin embargo, como observa Borges (1995: 14-15), no es una obviedad precisar la dirección del tiempo. Que del pasado fluye hacia el porvenir es la interpretación más común, pero no es menos razonable invertir esa lógica:

En la sombra la lluvia se diluye y en el silencio el son de la campana, nocturno el río de las horas fluye

desde su manantial, que es el mañana 
eterno, y en sus negras aguas huye aquella mi ilusión harto temprana.

El soneto de Unamuno ya nos ofrece una primera modificación conceptual, pues asocia el futuro (el 'por-venir') al agua nueva que nos llega desde la cabecera, desde la naciente del río y, consecuentemente, el pasado, al agua que pasa por nuestra ubicación y continúa su curso hacia el estuario. El elemento crucial que permite esta transformación de la dirección del flujo del tiempo es la ubicación del observador. Si situamos al observador como navegante que sigue el curso del río, el futuro se nos desvela como lo nuevo que de nosotros se aproxima, que se nos presenta y que por nosotros pasa, a medida que nos movemos en dirección a la desembocadura. Si, por el contrario, ubicamos al observador estáticamente a las márgenes del río, lo nuevo se nos aproxima desde el manantial y, en este sentido, el futuro emana.

Cabe prevenir, no obstante, que esta alternativa no conlleva un cambio de alineamiento entre pasado y futuro, por una parte, y delante y detrás, por otra. Pues en ambos casos el futuro está delante y el pasado va quedando atrás. La diferencia fundamental radica en si el observador navega, o sea, si es él el que se mueve en relación a los objetos de la orilla o, si, por el contrario, lo que se mueve son los objetos transportados por el flujo del agua. No se trata, pues, de una temporalidad aimara. Poéticay conceptualmente deriva de esta alternativa metafórica la diferencia entre un futuro con fin, un porvenir sin mucho futuro (Manrique) versus la eternidad del porvenir (Unamuno).

Otra posibilidad de variación poética tiene que ver con el medio en el que se produce el movimiento. La tradición luso-brasileña nos obsequia con un ejemplo de esta modificación metafórica que está dotada de una trascendencia existencial significativa. Cuando el navegante se aleja del estuario, se da cuenta que el flujo es incierto, que no hay una sola dirección, sino muchas (o ninguna) y que no es él el que se navega, sino que el que lo navega es el mar. Sin la fatalidad de un destino - al que ir derecho a se acabar y consumir - y sin dirección cierta, el navegante deja de ser timonero de sí mismo e incluso prescinde de la necesidad de serlo. Llegado este momento, lo verdaderamente necesario es navegar en el océano de la vida, en lugar de simplemente vivir anclado en un puerto o en el aburrimiento existencial de un cauce conocido (Pessoa). La letra de la samba Timoneiro de Paulinho da Viola es un caso paradigmático (y sin duda uno de los más populares) de esta variación:

$\begin{array}{ll}\text { Não sou eu quem me navega } & \text { No soy yo quien me navega } \\ \text { Quem me navega é o mar } & \text { Quien me navega es el mar } \\ \text { Não sou eu quem me navega } & \text { No soy yo quien me navega } \\ \text { Quem me navega é o mar } & \text { Quien me navega es el mar } \\ \text { É ele quem me carrega } & \text { Él es quien carga conmigo } \\ \text { Como nem fosse levar } & \text { Como si no me llevara } \\ \text { É ele quem me carrega } & \text { Él es quien carga conmigo } \\ \text { Como nem fosse levar } & \text { Como si no me llevara }\end{array}$

E quanto mais remo mais rezo

Y cuanto más remo más

Pra nunca mais se acabar

rezo

Essa viagem que faz

Sin conseguir terminar

O mar em torno do mar

Ese viaje que hace

El mar en torno del mar

\footnotetext{
${ }^{4}$ Agradezco enormemente a María del Mar Páramos Cebey por la traducción de los poemas en portugués brasileño. La traducción de los poemas en otras lenguas es de mi autoría.
} 
Meu velho um dia falou Com seu jeito de avisar:

- Olha, o mar não tem cabelos

Que a gente possa agarrar

$$
(\ldots)
$$

Timoneiro nunca fui Que eu não sou de velejar O leme da minha vida Deus é quem faz governar E quando alguém me pergunta

Como se faz pra nadar Explico que eu não navego Quem me navega é o mar

\section{(...)}

A rede do meu destino Parece a de um pescador Quando retorna vazia Vem carregada de dor Vivo num redemoinho Deus bem sabe o que ele faz A onda que me carrega Ela mesma é quem me traz
Mi viejo me dijo un día

Con su forma de avisar:

-Mira, el mar no tiene pelo

Para poderlo agarrar

\section{(...)}

Timonel yo nunca fui Porque no soy de navegar El timón de mi vida Dios lo hace gobernar Y cuando alguien me pregunta

Cómo se logra nadar Le explico que no navego Quien me navega es el mar

$$
(\ldots)
$$

La red de mi destino Parece la de un pescador Cuando retorna vacía Viene llena de dolor Vivo en un remolino Dios sabe bien lo que hace La ola que me carga Es la misma que me trae

¿Qué decir del tiempo en la mar? Probablemente, que no nos llegamos a hacer la ilusión de que sea eterno, pero que nos ilusiona y nos alivia mucho saberlo incierto. Pero volvamos por un momento al río, guiados esta vez por la pericia de gran un timonel de muchas travesías.

Eu vejo aquele rio a deslizar O tempo a atravessar meu vilarejo

E às vezes largo

$\mathrm{O}$ afazer

Me pego em sonho

A navegar

Com o nome Paciência

Vai a minha embarcação

Pendulando como o tempo

E tendo igual destinação

Pra quem anda na barcaça

Tudo, tudo passa

Só o tempo não

Passam paisagens furta-cor Passa e repassa o mesmo cais
Veo aquel río deslizarse

El tiempo atravesando mi

aldea

$\mathrm{Y}$ a veces abandono

Mi quehacer

Me deparo en sueños

Navegando

Con el nombre Paciencia

Va mi embarcación

Pendulando como el tiempo

Y con el mismo destino

Para quien anda en la barcaza

Todo, todo pasa

Menos el tiempo

Pasan los paisajes tornasolados Pasa y repasa el mismo muelle 


$\begin{array}{ll}\begin{array}{l}\text { Num mesmo instante eu vejo a } \\ \text { flor } \\ \text { Que desabrocha e se desfaz } \\ \text { Essa é a tua música }\end{array} & \begin{array}{l}\text { En un mismo instante veo la } \\ \text { flor } \\ \text { E tua respiração } \\ \text { Mas eu tenho só teu lenço } \\ \text { Em minha mão }\end{array} \\ \begin{array}{l}\text { Esa es tu música } \\ \text { Y tu respiración }\end{array} \\ \text { Pero yo solo tengo tu pañuelo } \\ \text { En mi mano }\end{array}$

En Xote de navegação, Chico Buarque realiza una amalgamación conceptual -un blending, en el sentido propuesto por Fauconnier y Turner (2002) - entre las dos metáforas básicas TIEMPO COMO MOVIMIENTO y TIEMPO COMO MOVIMIENTO DEL OBSERVADOR, aplicadas al caso concreto del río. Imaginariamente (en sueños o en ensueño), el observador estático abandona su ubicación en el margen del río y se lanza en aventuras a bordo de una barcaza, llevando consigo, no obstante, su condición inicial de inmovilidad. Al permanecer él inmóvil y con él el flujo del río, la única posibilidad de mantener el movimiento reside en transferirlo a los objetos de las márgenes: no es el barco el que navega hacia delante, sino que es la ribera la que navega para atrás. Al detener el flujo del río, se detiene también el paso del tiempo, representado por él en la metáfora original. Con ello, por y para el navegante estático todo pasa, menos el tiempo. En su barcaza, el tiempo se eterniza por un río con aguas infinitas, tan infinitas talvez como las del lago Biel, que mecen a su capricho y merced el bote de Rousseau, sin llevarlo, empero, a ningún lugar, a no ser a la eternidad del momento.

Aprovechemos que estamos hablando de Chico Buarque y veamos rápidamente un ejemplo más en el que el poeta utiliza una técnica de amalgamación conceptual.

Vivia a te buscar

Porque pensando em ti

Corria contra o tempo

Eu descartava os dias

Em que não te vi

Como de um filme

A ação que não valeu

Rodava as horas pra trás

Roubava um pouquinho

E ajeitava o meu caminho

Pra encostar no teu

Subia na montanha

Não como anda um corpo

Mas um sentimento

Eu surpreendia o sol

Antes do sol raiar

Saltava as noites
Vivía buscándote

Porque pensando en ti

Corría contra el tiempo

Y descartaba los días

En los que no te vi

Como de un film

La acción que no valió

Rodaba las horas hacia atrás

Robaba un poquito

Y arreglaba mi camino

Para acercarme al tuyo

Subía a la montaña

No como anda un cuerpo

Sino un sentimiento

Sorprendía el sol

Antes del amanecer

Saltaba las noches 
Sem me refazer

E pela porta de trás

Da casa vazia

Eu ingressaria

E te veria

Confusa por me ver

Chegando assim

Mil dias antes de te conhecer
Sin rehacerme

Y por la puerta de atrás

De la casa vacía

Me introduciría

Y te vería

Confusa por verme

Llegar así

Mil días antes de conocerte

El poema Valsa brasileira está estructurado a partir de una amalgamación entre el tiempo como objetos en movimiento que se acercan 'a su tiempo' y con su velocidad normal hacia el observador y un observador ansioso que no permanece simplemente inmóvil a la espera (como determina la metáfora TIEMPO COMO MOVIMIENTO), sino que se pone en movimiento, dirigiéndose impaciente a su encuentro, tan rápidamente que llega antes de que las cosas 'pasen'.

Para finalizar, reparemos en algunos casos que más que producir una alteración de las metáforas, concretizan alguno de los elementos constitutivos de sus estructuras.

Die Zeit überschlägt sich wie ein Stein vom Berge herunter, und man weiss nicht, wo sie hinkommt und wo man ist.

(Johann Wolfgang von Goethe)

Was verkürzt mir die Zeit? Tätigkeit!

Was macht sie unerträglich lang?

- Müssiggang!

(Johann Wolfgang von Goethe)
El tiempo se precipita como una piedra desde lo alto de una montaña y no sabemos a dónde va a caer ni dónde estamos nosotros.

(Johann Wolfgang von Goethe)

¿Qué me acorta el tiempo? ¡Actividad! ¿Qué hace con que sea insoportablemente largo? ¡Ociosidad! (Johann Wolfgang von Goethe)

Dreifach ist der Schritt der Zeit: Zögernd kommt die Zukunft hergezogen, Triple es el paso del tiempo: vacilante se va acercando el Pfeilschnell ist das Jetzt vuela el presente; entflogen, $\quad$ y eternamente en calma queda el Ewig still steht die pasado. Vergangenheit.

(Friedrich von Schiller)

\section{(Friedrich von Schiller)}

El primer ejemplo de Goethe no modifica la estructura básica de la metáfora TIEMPO COMO MOVIMIENTO, pero concretiza la manera de la aproximación de los objetos que representan el tiempo, indicando que, más que de un suave y previsible acercamiento, se trata de una precipitación abrupta. El tiempo y sus acontecimientos se nos vienen (o se nos echan) encima, sin que podamos experimentar la posibilidad de 'tener el tiempo' necesario de percibir su aproximación.

Los dos versos subsiguientes de Goethe y los de Schiller tematizan igualmente nuestra percepción del paso del tiempo. El tiempo se nos hace insoportablemente lento si 
no nos ocupamos de otra cosa a no ser de él mismo y, por el contrario, no lo vemos ni pasar, si nos mantenemos activamente ocupados con otras cuestiones. Ya en los versos de Schiller, la diferencia de percepción de la velocidad del paso del tiempo se asocia a los propios momentos básicos de la temporalidad: un futuro que se nos acerca titubeante, un presente fugaz y un pasado que reposa eternamente. Una caracterización análoga -sea dicho de paso-nos la ofrece Schopenhauer en sus Aforismos (1949: 223-224), en relación con las fases o momentos de la vida, cuando señala que, desde el punto de vista de la juventud, la vida es un futuro interminable y el tiempo transcurre a pasos lentos, mientras que en los años otoñales percibimos que el pasado fue un soplo y que el tiempo pasa volando.

El próximo es quizás uno de los más bellos poemas-canción de Joan Manuel Serrat y un bello ejemplo también de la utilización del concepto de destiempo. Este concepto presupone y reafirma la existencia de un flujo normal y correcto del tiempo de las cosas y de los acontecimientos que sirve como tela de fondo para situar la existencia de un elemento que no se atiene a él, que transgrede la sucesión normal, que se empecina a nadar a contracorriente y que, a pesar de estar condenado a la más fugaz brevedad, aunque sea por tan solo un momento, consigue que se detenga el tiempo, sorprendiéndonos y alegrándonos, como aquel inesperado último beso de un amor que, en realidad, sabemos que ya ha acabado.

La llarga nit de l'hivern s'ha encès quan de sotamà el roser ha fet una rosa més vermella que la sang.

Darrer sospir de l'estiu. Penyora de la bonança. Ocell que ha caigut del niu.

Festeja amb un raig de sol arrecerada a un xiprer; ¿fas tard o véns massa d'hora, meravellós malentès?

Nascuda contra corrent desafiant les gelades i plantant-li cara al vent.

Com una flor de paper no escoltaràs el zum-zum de les abelles xuclant-te ni escamparàs cap perfum.

Mai no et regalarà el sol la son de les migdiades ni els capvespres de juliol.

Però abans no t'escanyin les impacients mans de l'hivern la tarda et mira i s'alegra, es detura $i$ es sorprèn amb una petita $\mathrm{i}$ breu
La larga noche del invierno se ha encendido cuando bajo mano el rosal hizo una rosa más roja que la sangre.

Último suspiro del verano

Prenda de la bonanza

Pájaro caído del nido.

Festeja con un rayo de sol abrigada por un ciprés ¿Haces tarde o vienes demasiado temprano, maravilloso malentendido?

Nacida a contracorriente desafiando las heladas y plantándole cara al viento.

Como una flor de papel No escucharas el zumbido de las abejas chupándote ni esparcirás ningún perfume.

El sol nunca te regalará el sueño de las siestas ni los atardeceres de Julio.

Pero antes de que te ahoguen Las impacientes manos del invierno la tarde te mira y se alegra, se detiene y se sorprende. 
-talment la flor dels teus llavisrosa roja de l'adéu.
Con una pequeña y breve -como la flor de tus labiosrosa roja del adiós...

Finalizamos nuestro recorrido, como casi no podría ser diferente, acompañando por un instante al caminante de Machado 5 .

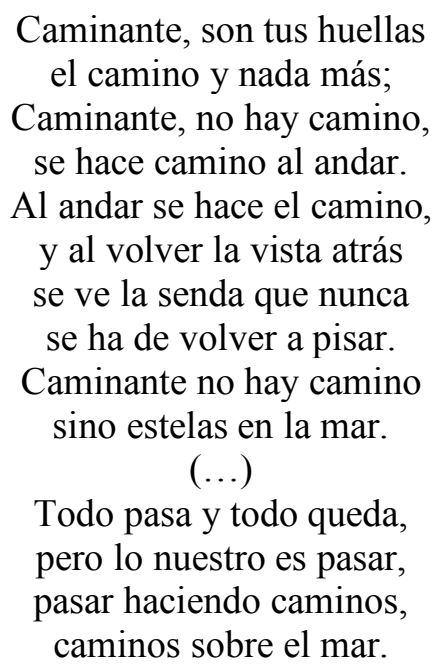

Construido sobre la metáfora general TIEMPO COMO MOVIMIENTO DEL OBSERVADOR (sujeto), este poema machadiano crea una ansiedad existencial a partir de dos elementos esenciales de la metáfora, a saber, (i) la irreversibilidad del tiempo, en el sentido heracliano, según el cual es imposible bañarse dos veces en el mismo río y (ii) el determinismo y la fatalidad del destino, según los cuales el ser humano iría inevitablemente cumpliendo tan solo un itinerario preexistente que lo llevaría inexorablemente al desenlace fatal de la muerte o, como alternativa no menos angustiante, estaría vagando por un mundo sin caminos. Machado contrarresta la ansiedad derivada de estos elementos mediante dos concretizaciones de la metáfora original. Frente a un camino con puntos predeterminados, por los que tenemos que ir pasando, o la falta de camino, Machado contrapone un apelo a la conciencia sobre el valor de nuestros actos, como salida a ambas vicisitudes: el camino no nos es dado, pero podemos evitar perdernos en un mundo sin caminos, porque somos capaces de hacer el nuestro propio. No hay camino, pero se hace camino al andar. En la estructura de la metáfora, esto supone una fusión entre el sujeto (caminante) y el itinerario (camino), en el sentido de que este último resulta, se va creando, de las acciones del primero. A esta fusión estructural se une una segunda, necesaria para desafiar la transitoriedad e irreversibilidad y, a final de cuentas, el sentimiento de pérdida, inherentes al paso del tiempo. Se trata de la fusión entre los tiempos, es decir, entre los espacios temporales diferenciados en la metáfora. A la estricta secuencialidad y unidireccionalidad impuestas por la metáfora de origen espacial se opone una coocurrencia de los tiempos vividos, en la que cada momento presente está constituido por una presencia del pasado (todo pasa y todo queda) que proyecta el futuro (pero lo nuestro es pasar): un hoy de ayer que proyecta un mañana.

\footnotetext{
${ }^{5}$ La bibliografía sobre el tiempo y la temporalidad en la poesía machadiana en el ámbito de los estudios literarios es bastante amplia. Señalo aquí a Caparrós Esperante (1997) como ejemplo y para tener acceso a más referencias bibliográficas de dicho ámbito.
} 
Esta amalgamación de la temporalidad en cada presente actual -sintetizada con enorme fuerza expresiva en su famoso aforismo Hoy es siempre todavía-constituye, sin duda, la respuesta privilegiada por Machado a su inquietud filosófica en torno a la temporalidad y, consecuentemente también, un procedimiento lírico muy frecuente en su producción poética. Valgan, para finalizar, dos breves ejemplos más de la utilización de este recurso.

Esta luz de Sevilla... Es el palacio donde nací, con su rumor de fuente.

Mi padre, en su despacho. - La alta frente, la breve mosca, y el bigote lacio-.

Mi padre, aun joven. Lee, escribe, hojea sus libros y medita. Se levanta; va hacia la puerta del jardín. Pasea. A veces habla solo, a veces canta.

Sus grandes ojos de mirar inquieto ahora vagar parecen, sin objeto donde puedan posar, en el vacío.

Ya escapan de su ayer a su mañana; ya miran en el tiempo, ipadre mío!, piadosamente mi cabeza cana.

Hoy, con la primavera, soñé que un fino cuerpo me seguía cual dócil sombra. Era mi cuerpo juvenil, el que subía de tres en tres peldaños la escalera.

- Hola, galgo de ayer. (Su luz de acuario

Trocaba el hondo espejo

Por agria luz sobre un rincón de osario.)

- ¿Tú conmigo, rapaz?

- Contigo, viejo.

El espacio poético del soneto vence la fatalidad del tiempo y propicia el encuentro entre el padre joven y su hijo viejo. Esta simultaneidad de presentes distantes requiere desde la perspectiva del padre- una fusión entre su presente y el futuro y -desde la visión del hijo- entre su presente y el pasado. El mismo procedimiento de fusión actúa también en el segundo poema, permitiendo, en este caso, el desdoblamiento del ser en un Yo-antes $\mathrm{y}$ un Yo-ahora $\mathrm{y}-\mathrm{y}$ en esto reside la virtuosidad del procedimiento- su concurrencia en el presente actual, su reunificación en un 'Yo-ahora-que-es-un-antes-todavía'.

En la próxima sección veremos que la fusión de tiempos es también un procedimiento fundamental de la reflexión fenomenológica. Antes de adentrarnos en el campo filosófico me parece útil, no obstante, que hagamos una síntesis de los tipos de variación metafórica que hemos visto hasta ahora.

De acuerdo con lo que hemos visto hasta ahora, podemos constatar, en primer lugar, variaciones que afectan, de forma inmediata, al dominio fuente (Source) de las metáforas. El dominio fuente puede variar con relación a la base experimental concreta de la metáfora. En algunos casos (la mayoría, en nuestros ejemplos), la base está constituida por la experiencia de navegar, mientras que en otros (en Machado) por la de caminar o andar. Además, la experiencia de navegar se puede llevar a cabo en el río o en 
el mar. Existe, pues, una diferenciación del medio en el que se produce el movimiento, manteniéndose inalterado el resto de los elementos constitutivos del espacio fuente (navegante, embarcación, etc.). Otra alteración resulta de la introducción de un elemento específico en el espacio fuente, que sobresale a los elementos genéricos que default integran este espacio. Es lo que sucede con la rosa a destiempo en la canción de Serrat o el Yo impaciente en la de Chico Buarque. Finalmente, tenemos casos en los que no se introduce un nuevo elemento, sino que se concretiza uno ya presente, como ocurre con el tipo de movimiento y su velocidad en los versos de Goethe y Schiller, así como en los aforismos de Schopenhauer. Cabe puntualizar, obviamente, que, aunque estas variaciones afecten directamente al espacio fuente, sus consecuencias conceptuales se propagan al espacio meta, es decir, a la forma como se conceptúa filosófica o poéticamente el tiempo, como lo hemos ido viendo a lo largo de las páginas precedentes.

Un segundo tipo de variación es el que afecta a la conexión (mapping) entre elementos del espacio fuente y del espacio meta. Es lo que sucede con la asociación entre la cabecera y el curso inicial de un río, por una parte, y el curso final y la desembocadura, por otra, como elementos del espacio fuente, con futuro y el pasado, como elementos del espacio meta. Vimos que los versos de Unamuno conectan la cabecera y el curso inicial al futuro y el curso final la desembocadura al pasado, mientras que Las Coplas de Jorge Manrique (así como la mayoría de los otros poemas) hacen lo contrario. No está por más decir aquí también que estas alternativas estructurales repercuten en la conceptuación poética del tiempo y de la temporalidad.

El último tipo de variación constatado es el que deriva de procesos de fusión o amalgamación (blending), para usar el término más técnico. Este tipo se divide en dos subtipos: (i) cuando el proceso de amalgamación tiene lugar entre las dos metáforas generales del tiempo (o elementos pertenecientes a cada una de ellas). Es lo que sucede, por ejemplo, en los poemas Xote de Navegação y Valsa Brasileira, de Chico Buarque. Y (ii), cuando la amalgamación se produce entre elementos de la misma metáfora, como observamos en los poemas de Antonio Machado.

A continuación, y para finalizar la sección, veremos que la amalgamación también desempeña un papel central en la reflexión fenomenológica sobre el tiempo. Escogemos como texto base para el análisis el capítulo La Temporalité de la Phénomenologie de la Perception (1945) de Maurice Meleau-Ponty, que a su vez se fundamenta en la teoría de la estructura temporal de la consciencia de Edmund Husserl, a la que nos referimos a continuación a partir de Husserl (2002).

Merleau-Ponty parte también de la metáfora del río para iniciar sus reflexiones, pero advierte inmediatamente que en sí el flujo de un río no posee ningún elemento que remita a la temporalidad. Para ello, se torna imprescindible la presencia de un observador que adopte un punto de vista e imponga una «visión sobre el tiempo» (1945: 472). Los dos puntos de observación lógicos - como navegante que sigue el curso del río o ubicado en las márgenes - nos proporcionarán, argumenta Ponty, experiencias diferentes de las que resultan, consecuentemente, visiones distintas sobre el tiempo. En el segundo caso, las aguas que pasan por el observador se van dirigiendo al pasado, mientras que el futuro se encuentra en la cabecera y el curso inicial del río. En el primero, por el contrario, el observador-navegante se dirige con el propio curso del río hacia su futuro, representado por los objetos que va encontrando por las márgenes y, por la propia desembocadura («que es el morir»). Como vemos, pues, Merleau-Ponty parte de las dos metáforas generales de la temporalidad concretizadas en el río como base experimental.

El autor concluye de estas reflexiones iniciales que «el tiempo no es un proceso real, una sucesión efectiva que yo me limitaría a registrar, sino que más bien nace de mi relación con las cosas» (1945: 473). Esto es, Merleau-Ponty transfiere el tiempo y la 
temporalidad de las cosas en sí a nosotros mismos, a nuestra consciencia. Ahora bien, al mismo tiempo puntualiza que esta transferencia no debe ser entendida como si la consciencia dispusiera de un mecanismo, de una especie de vía, que nos permitiese acceder objetivamente al pasado y mucho menos al futuro. En este sentido, refuta que yo pueda tener acceso a la consciencia que tuve de un hecho terminado del pasado a partir de un rasgo psicológico que conservo en mi memoria, pues este rasgo es algo presente y no una suerte de máquina del tiempo que me pudiese transportar de nuevo a mi consciencia de aquel hecho (Merleau-Ponty, 1945: 474-475).

Para caracterizar adecuadamente la temporalidad como estructura de nuestra consciencia, Merleau-Ponty recurre a Husserl. Como es sabido, según este autor, la estructura temporal de la consciencia está constituida por la síntesis actual, es decir en cada momento presente, de tres dimensiones fundamentales: impresión primaria, retención y protención (Husserl, 2002: 80-165). La impresión primaria (Urimpression) es el objeto que corresponde a mi consciencia actual, lo experimentado o percibido ahora, en este momento: el objeto que centra mi atención en este instante, la nota actual de una melodía, el enunciado que estás profiriendo, etc. (Husserl, 2002: 95-100; Merleau-Ponty, 1945: 478-491). Según Husserl, es imposible que las impresiones primarias ocurran de forma aislada, sino que van siempre acompañadas e indisociablemente entrelazadas con elementos pertenecientes a las otras dos dimensiones de la consciencia, a saber, elementos retencionales y protensionales. La dimensión retencional nos proporciona la consciencia sobre el objeto que acaba de dejar de ser el centro de nuestra atención (Husserl, 2002: 100-106). En la retención se ancla, se enraíza mi intención primaria actual, atribuyéndole el contexto temporal inmediato para su interpretación. La nota que acabo de oír y cuya materialidad acústica ya se ha desvanecido continúa presente como el contexto de consciencia y el horizonte de sentido inmediatos en los que se enraíza la nota que se está produciendo en este preciso momento. Del mismo modo y simultáneamente, la nota actual, semantizada en el contexto de sus retenciones, crea una expectativa de cómo va a seguir la melodía que estamos oyendo. Es decir, anticipamos las probables futuras notas como un aspecto más que se entreteje con los otros dos para crear, en conjunto, nuestra conciencia actual. Es lo que Husserl denomina dimensión protensional (Husserl, 2002: 117-125).

En suma, el tiempo, ahora como estructura de nuestra consciencia y no como fenómeno preexistente en el mundo, deja de ser un mero flujo o una simple sucesión de objetos que se acercan a mí, pasan por mí y se van distanciando detrás de mi (o a los que yo me acerco, etc.). La Fenomenología abandona la idea de la secuencialidad y unidireccionalidad inherentes a las metáforas generales del tiempo y concretizadas en la base experimental del río y la substituye por el concepto de «síntesis» (Merleau-Ponty, 1945: 481), de entrelazamiento o amalgamación constantes, en cada momento actual, entre presente, pasado y futuro. Se observa con facilidad una fuerte analogía entre esta idea de fusión temporal y la elaborada en la poética de Machado. También la Fenomenología transforma las metáforas generales del tiempo mediante la aplicación de mecanismo de blending a la estructura interna de las mismas, para integrar conceptualmente tres elementos, originalmente diferenciados, en una sola unidad fusionada. Obviamente, cabe acrecentar que la reflexión fenomenológica nos proporciona una elaboración pormenorizada y escrupulosa de cómo se produce este relacionamiento e integración conceptual, que aquí solamente hemos podido reproducir de forma muy sucinta (y que la poesía condensa con su enorme poder expresivo en fórmulas como Hoy es siempre todavía). 


\section{CONCLUSIONES}

Nos preguntábamos al inicio de este texto si los conceptos culturales de la temporalidad influencian nuestras reconceptualizaciones estéticas y filosóficas o si éstas últimas, por el contrario, consiguen liberarse de los primeras. Considerando el análisis que hemos desarrollado a lo largo de la última sección, es aconsejable una respuesta ponderada. Pues, por una parte, queda patente que, independientemente del grado de complejidad que adquieren los conceptos poéticos y filosóficos del tiempo, en ninguno de los casos estudiados trascienden su enraizamiento en las metáforas generales. Hasta en los casos de mayor complejidad, como en Machado o en Merleau-Ponty y Husserl, el punto de partida continúa estando constituido por una o las dos metáforas TIEMPO COMO MOVIMIENTO O TIEMPO COMO MOVIMIENTO DEL SUJETO. Estas metáforas parecen actuar como anclas, como estructuras de pensamiento que preestablecen lo que puede o no puede ser concebido sobre el fenómeno de la temporalidad. Adicionalmente, el análisis sugiere también una preponderancia del río como base experimental concreta $y$, consiguientemente, también como fundamento para reelaboraciones. En ningún caso, los conceptos complejos plantean conceptuaciones del tiempo que presenten un distanciamiento cultural y se aproximen, por ejemplo, a visiones sobre el fenómeno como las propias de la cultura china, aimara o tupí, a las que hemos hecho mención en la sección 2. Queda claro, pues, de un modo general, el anclaje cultural del pensamiento complejo.

Por otra parte, sin embargo, también queda evidente que las metáforas generales son el punto de partida para la producción de variaciones metafóricas que pueden alcanzar altos grados de complejidad. En la mayoría de los casos que hemos visto en el presente trabajo, estas variaciones van más allá del nivel del lenguaje. Es decir, no se trata tan sólo de nuevas formas lingüísticas para expresar las mismas metáforas inalteradas, sino de la construcción de metáforas temporales con estructuras conceptuales propias. En otras palabras: la poesía y el pensamiento filosófico conceptualizan metafóricamente la temporalidad en procesos que tienen como dominio meta la experiencia de la temporalidad (proto-temporalidad) y como dominio fuente las metáforas generales TIEMPO COMO MOVIMIENTO O TIEMPO COMO MOVIMIENTO DEL SUJETO.

Como expusimos en la sección anterior, las variaciones pueden ser clasificadas de acuerdo con el elemento de la metáfora general afectado de una forma más inmediata, así como el procedimiento usado para producir la modificación. Reproducimos a continuación sintéticamente la clasificación obtenida:

(1) Variación en el dominio fuente.

- Variación en la base experimental.

- Introducción de un elemento específico en el dominio fuente.

- Concretización de un elemento ya existente en el dominio fuente.

(2) Variación en las conexiones (mappings) entre espacio fuente y espacio meta.

(3) Variación por amalgamación (blending).

- Amalgamación intermetafórica (entre elementos de distintas metáforas).

- Amalgamación intrametafórica (entre los elementos de la misma metáfora).

Con respecto a esta clasificación, merece la pena hacer dos breves observaciones. En primer lugar, cabe señalar que los tipos de variación y los mecanismos constatados no 
difieren de los descritos en estudios sobre la variación intercultural de metáforas, independientemente de los dominios experimentales o conceptuales involucrados. Así, los tres tipos de variación son registrados, por ejemplo, en Kövecses (2005: 117-130). En el caso de la variación por amalgamación, no obstante, el subtipo intrametafórico no aparece en la clasificación de este autor. En segundo lugar, los casos analizados en este trabajo muestran que los conceptos más complejos, aquellos que manifiestan mayor grado de elaboración y que, consiguientemente, varían más en relación con las metáforas generales son obtenidos mediante operaciones de amalgamación conceptual. Esto comprueba, una vez más, el potencial de este mecanismo como generador de pensamiento de alta complejidad (Turner, 2014). Merecen una mención especial, en este sentido, las (re-)conceptualizaciones filosófico-poéticas machadianas y el concepto fenomenológico de la temporalidad. El paralelismo entre ambos no se debe únicamente a la aplicación (intrametafórica) del mecanismo de la amalgamación conceptual, sino que abarca también el resultado obtenido, a saber, la síntesis constante (encada momento presente) entre pasado, presente y futuro.

Finalizo con una intuición más general: las variaciones que hemos estudiado, sobre todo las poéticas, parecen responder (entre otras cosas, seguramente) a una motivación común: reconsideraciones semánticas, puntos de fuga que, por lo menos, mitiguen las ansiedades que puedan emanar de una linealidad y una irreversibilidad del tiempo y una fatalidad del destino, inmanentes en nuestra concepción cultural de la temporalidad. Una resistencia obstinada, como brillantemente expresa Rubén Darío en su Canción de otoño en primavera:

Mas a pesar del tiempo terco, mi sed de amor no tiene fin; con el cabello gris, me acerco a los rosales del jardín...

\section{REFERENCIAS BIBLIOGRÁFICAS}

Borges, Jorge Luis. 2005. Historia de la eternidad. Madrid: Alianza Editorial.

Caparrós Esperante, Luis. 1997. «Hoy es siempre todavía: la plasticidad del ayer en los poemas de Antonio Machado». En Ann Mackenzie (ed.), Spain and its Literature. Essays in Memory of E. Allison Peers. Liverpool: Liverpool University Press, pp. 317-345.

Depraz, Natalie. 2001. «The Husserlian Theory of Intersubjectivity as Alterology: Emergent Theories and Wisdom Traditions in the Light of Genetic Phenomenology». En Between Ourselves. Second-person issues in the study of consciousness, ed. Evan Thompson. Charlottesville: Imprint Academic, pp. 169178.

Ellen, Roy. 2016. «The Cultural Cognition of Time. Some Anthropological Perspectives». En Conceptualizations of Time, ed. Barbara LewandowskaTomaszczyk. Amsterdam: John Benjamins, pp. 125-149.

Fauconnier, Gilles y Mak Turner. 2002. The Way we Think. Conceptual Blending and the Mind's Hidden Complexities. New York: Basic Books.

Fuchs, Peter. 1995. Die Umschrift. Zwei kommunikationstheoretische Studien: japanische Kommunikation und Autismus. Frankfurt am Main: Suhrkamp.

Heine, Bernd y Tania Kuteva. 2001. World Lexicon of Grammaticalization. Cambridge: Cambridge University Press.

Huelva Unternbäumen, Enrique. 2013. Intersubjetividad y gramática. Aspectos de una gramática fenomenológica. Frankfurt am Main: Peter Lang. 
Husserl, Edmund. 2002. «Phänomenologie des inneren Zeitbewußseins». En Phänomenologie der Lebenswelt, Ednund Husserl. Stuttgart: Reclam.

Kövecses, Zoltán. 2005. Metaphor in Culture. Universality and Variation. Cambridge: CUP.

—. 2008. «Universality and Variation in the Use of Metaphor». En Selected Papers from the 2006 and 2007 Stockholm Metaphor Festivals, eds. Nils-Lennart Johanesson y David C. Minugh. Stockholm: Stockholm University, pp. 51-74.

- 2010. Metaphor. A Practical Introduction. Oxford: OUP.

-. 2011. «Methodological issues in conceptual metaphor theory». En Windows to the Mind: Metaphor, Metonymy and Conceptual Blending, eds. Sandra Handl Y Hans-Jörg Schmid. Berlin y New York: Walter de Gruyter, pp. 23-39.

—. 2017. «Levels of metaphor». Cognitive Linguistics, 28(2): 321-347.

- 2018. Metaphor Universals in Literature. Manuscript. Budapest: Budapest University.

https://www.researchgate.net/publication/322940750_Metaphor_universals_in_1 iterature

Lakoff, George. 1993. «The contemporary theory of metaphor». En Metaphor and Thought, ed. Andrew Ortony. Cambridge: CUP.

Lakoff, George y Mark Johnson. 1980. Metaphors we live by. Chicago: Chicago University Press.

-. 1999. Philosophy in the Flesh. The Embodied Mind and its Challenge to Western Thought. New York: Basic Books.

Lakoff, George y Mark Turner. 1989. More than Cool Reason. A Field Guide to Poetic Metaphor. Chicago: Chicago University Press.

Luhmann, Niklas. 1995. «Zeit und Handlung. Eine vergessene Theorie». En Soziologische Aufklärung 3. Soziales System, Gesellschaft, Organisation, Niklas Luhmann. Opladen: Westdeutscher Verlag, pp. 101-125.

Merleau-Ponty, Maurice. 1945. Phénoménologie de la perception. Paris: Gallimard.

Núñez, Rafael. 2003. Conceptual Structures and Cultural Variation - Metaphorical Spatial Construals of Time in Aymara (Manuscript).

Núñez, Rafael y Eve Sweetser. 2006. With the Future behind Them. Convergent Evidence from Aymara Language and Gesture in the Crosslinguistic Comparison of Spatial Construals of Time. Cognitive Science, 30: 401-450.

Pragglejaz Group. 2007. «MIP: A Method for Identifying Metaphorically Used Words in Discourse». Metaphor and Symbol, 22(1): 1-39.

Raden, Günter. 2003. «The Metaphor TIME AS SPACE across Languages». En Übersetzen, interkulturelle Kommunikation, Spracherwerb und Sprachvermittlung - das Leben mit Sprachen, Festschrift für Juliana House zum 60. Geburstag. Zeitschrift für interkulturellen Frendsprachenunterricht, $8(2,3)$ : 226-239.

Shimohara, Kazuko. 2000. Up-down Orientation in Time Metaphors: Analyses of English and Japanese. Manuscrito. Tokyo: Tokyo University of Agriculture and Technology.

Rousseau, Jean-Jacques. 2012. Les rêveries du promeneur solitaire. En Collection complète des oeuvres, Genève, 1780-1789, vol. 10. www.rousseauonline.ch

Schopenhauer, Arthur. 1949. Aphorismen zur Lebensweisheit. Stuttgart: Reclam.

Sinha, Chris y Vera da Silva Sinha, Jörg Zinken, Wang Sampaio. 2011. «When Time is not Space: The social and linguistic construction of time intervals and temporal event relations in an Amazonian culture». Language and Cognition, 3(1): 137169. 
Sloterdijk, Peter. 2011. Stre $\beta$ und Freiheit. Frankfurt am Main: Suhrkamp.

Turner, Mark. 2014. The Origin of Ideas. Blending, Creativity and the Human Spark. Oxford: OUP.

Weinrich, Harald. 2001. Sprache, das heißt Sprachen. Tübingen: Gunter Narr.

Yu, Ning. 1998. The Contemporary Theory of Metaphor: A Perspective from Chinese. Amsterdam: John Benjamin. 